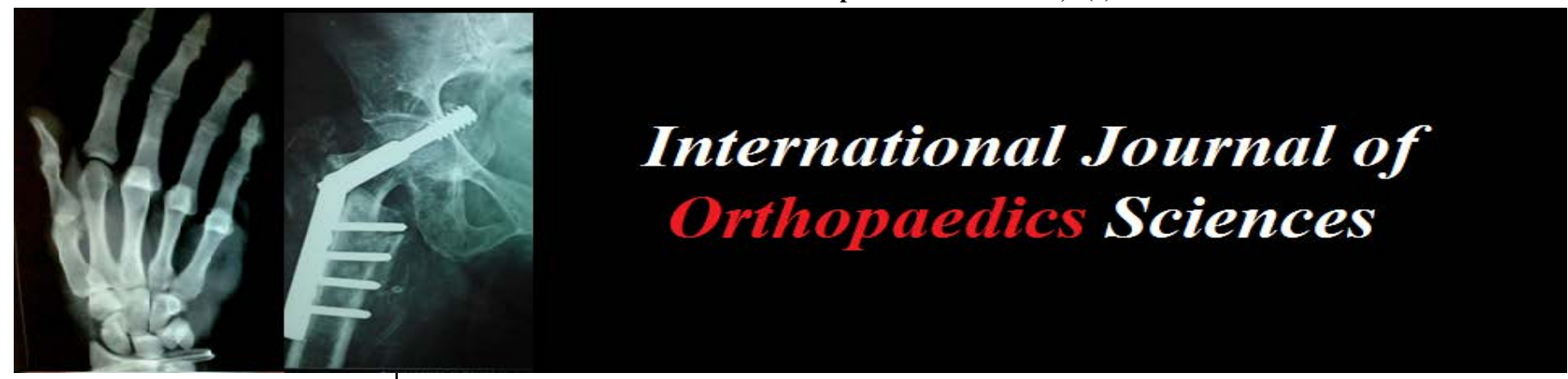

E-ISSN: 2395-1958

P-ISSN: 2706-6630

IJOS 2020; 6(4): 516-518

(C) 2020 IJOS

www.orthopaper.com

Received: 04-08-2020

Accepted: 09-09-2020

Dr. Afreen Ameer Hussain

Manchester Royal Infirmary,

Manchester University

Foundation Trust, United

Kingdom

Dr. Nishaanth R

Postgraduate, Department of Orthopaedics, Yenepoya Medica College, Mangalore, Karnataka, India

Dr. Abhishek V Shetty

Associate Professor, Department of Orthopaedics, Yenepoya

Medical College, Mangalore,

Karnataka, India
Corresponding Author: Dr. Abhishek V Shetty Associate Professor, Department of Orthopaedics, Yenepoya Medical College, Mangalore, Karnataka, India

\section{Outcome of limb salvage surgery in sarcomas around knee with mega endoprosthetic reconstruction}

\author{
Dr. Afreen Ameer Hussain, Dr. Nishaanth R and Dr. Abhishek V Shetty
}

DOI: https://doi.org/10.22271/ortho.2020.v6.i4h.2379

\section{Abstract}

Background: Currently $90-95 \%$ of sarcomas around the knee can be treated safely with wide resection and limb salvage surgery. Advancement of material science and design in endoprosthesis enables immediate rehabilitation, provides durable and functional limb. Primary goal is to restore and maintain stability and ambulation.

Aim of the study: To determine the functional outcome and complications of mega endoprosthesis.

Methods and Materials: Retrospective study conducted between 2008 to 2013 in an age group of 16 to 30 years with sarcomas around knee treated by mega endoprosthetic reconstruction. Total of 20 cases included in this study (Osteosarcoma- 12 cases, Giant cell tumour- 6 cases and Metastatic lesion - 2 cases) with tumour lesion at distal femur in 15 cases and 5 cases in proximal tibia. Follow up period was 48 months. Neo-adjuvant chemotherapy and post-op chemotherapy was given in selected cases. Functional outcome was scored by Musculoskeletal tumour society scoring (MSTS).

Results: The average MSTS functional score was $75 \%$ (Range - $60 \%$ to 100\%). 4-year survival rate was $100 \%$ in our study. Range of movements at 4 years was $0-90^{\circ}$. Complications was observed in 5 cases. 2 cases had foot drop, one case had knee dislocation, and one case had flap necrosis and aseptic loosening in one case.

Discussion: Individual approach to every patient is essential in order to choose the most suitable surgical treatment.All our patients were below 30 years and $2 / 3^{\text {rd }}$ were below 25 years of age, hence limb salvage is preferred over amputation.

Conclusion: Mega endoprosthetic reconstruction provides good functional outcome in patients with bone tumours around the knee. Endoprosthesis should be considered as treatment of choice for bone tumours around the knee.

Keywords: Limb salvage surgery, sarcoma around knee, mega endoprosthetic reconstruction

\section{Introduction}

Limb salvage surgery, currently an accepted bone tumour treatment method. It has been traditionally a difficulty in Orthopaedic oncology. The outlook of management of malignant tumours has greatly improved, and this has encouraged surgeons to consider limb salvage surgery in most cases. Improvement in the reconstructive techniques and the development of effective adjuvant chemotherapy for primary tumours made limb salvage surgery a feasible option ${ }^{[1,2]}$. But the long-term durability of various reconstructive methods is not known. Currently $90-95 \%$ of sarcomas around the knee can be treated safely with wide resection and limb salvage surgery ${ }^{[3-5]}$. Advancement of material science and design in endoprosthesis enables immediate rehabilitation, provides durable and functional limb. Primary goal is to restore and maintain stability and ambulation. Endoprosthetic replacement offers several advantages like shorter operating time, early stability, weight bearing mobilization and hospital stay in comparison to biological reconstructions. It also allows the early introduction of postoperative adjuvant therapy.

\section{Methods and Materials}

It is a Retrospective study. Study was conducted between 2008-2013. Patients with sarcomas around knee with an age group of 16 to 30 years which was treated by mega endoprosthetic reconstruction was included in this study. 
Total of 20 cases was included (Osteosarcoma - 12 cases, Giant cell tumour - 6 cases and Metastatic lesion - 2 cases) with tumour lesion at distal femur in 15 cases and 5 cases in proximal tibia. Patients was followed up for a period of 48 months. Neo-adjuvant chemotherapy and post-op chemotherapy was given in selected cases. Functional outcome was scored by Musculoskeletal tumour society scoring (MSTS).

Table 1: Showing the malignancy, number of cases and their site of location

\begin{tabular}{|c|c|c|c|}
\hline Malignancy & $\mathbf{n}=\mathbf{2 0}$ cases & Site & $\mathbf{n}=\mathbf{2 0}$ cases \\
\hline Osteosarcoma & 12 cases & Distal femur & 15 cases \\
\hline Giant cell tumour & 6 cases & Proximal tibia & 5 cases \\
\hline Metastatic lesion & 2 cases & & \\
\hline
\end{tabular}

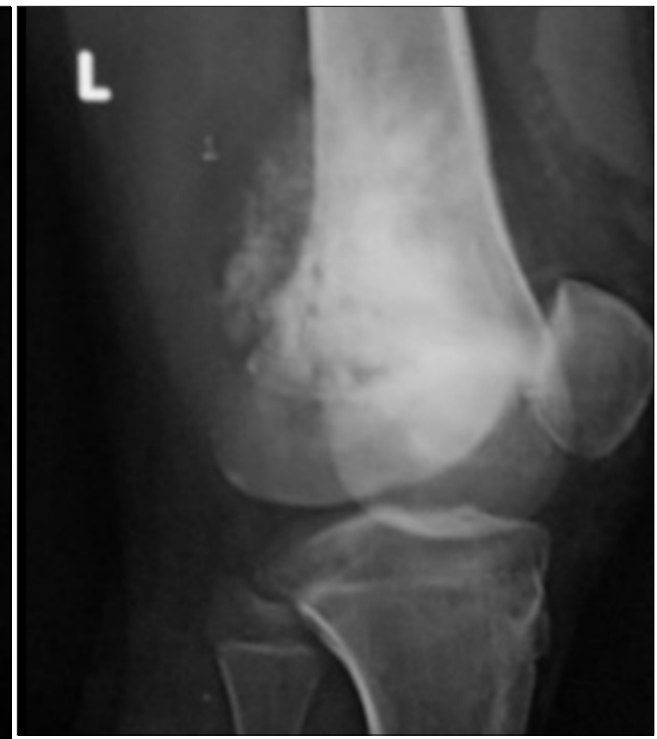

Fig 1: Showing the preoperative radiograph of 16 year old male child with osteosarcoma left distal femur

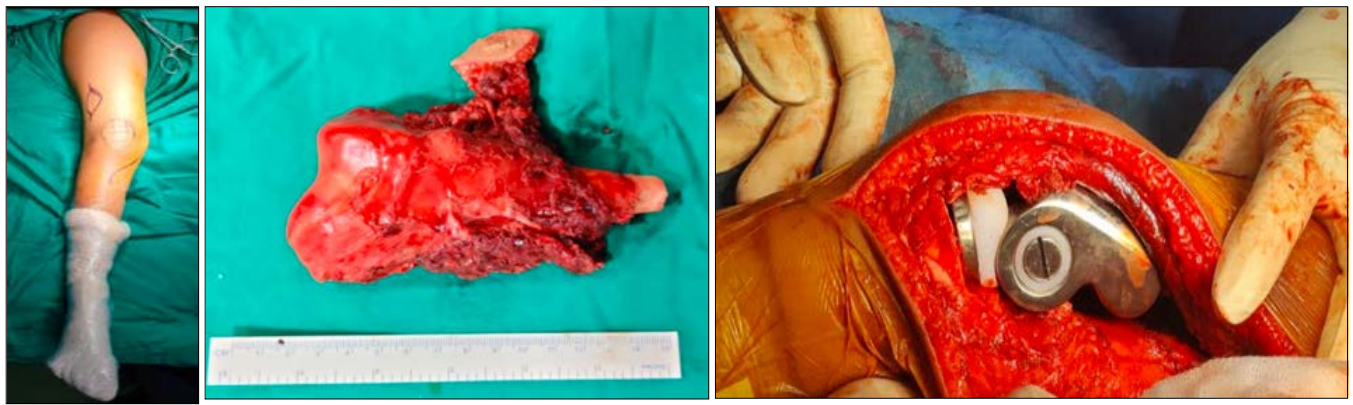

Fig 2: Showing incision for the wide excision of the tumor, excised specimen and prosthesis placement of 16 year old male child

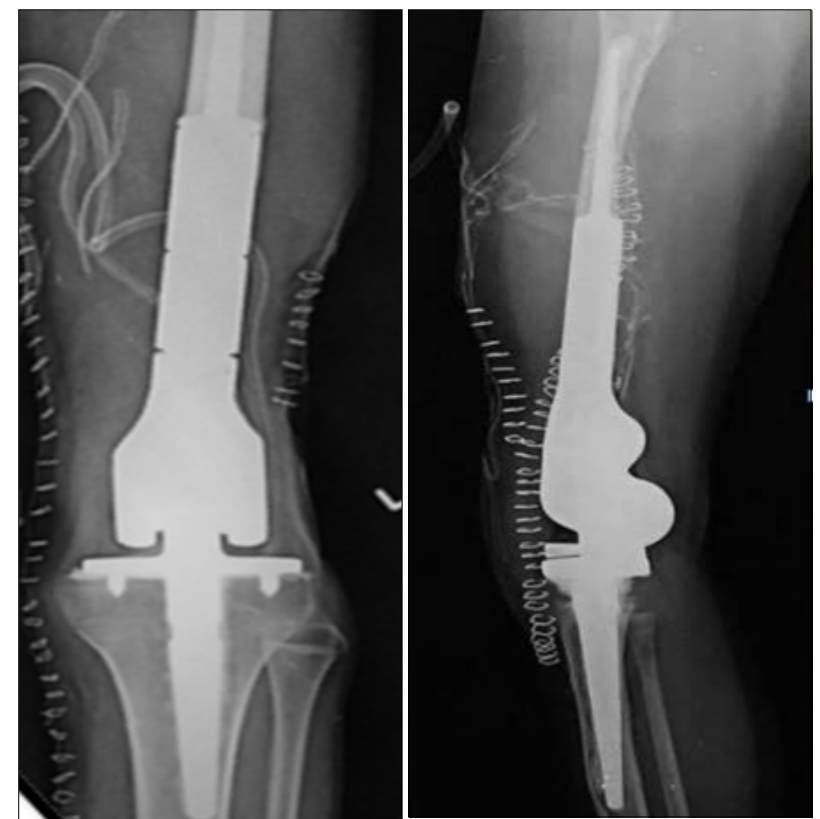

Fig 3: Showing postoperative radiograph with mega endoprosthesis for left distal femur and proximal tibia of 16 year old male child 


\section{Results}

Musculoskeletal tumour society scoring (MSTS) was used to assess the functional outcome of patients undergone limb salvage surgery with mega endoprosthesis at final follow up. The average MSTS functional score was 75\% (Range - 60\% to $100 \%$ ). 4-year survival rate was $100 \%$ in our study. Range of movements at 4 years was $0-90^{\circ}$. Postoperative complications was observed in 5 cases. 2 cases had foot drop, one case had knee dislocation, and one case had flap necrosis and aseptic loosening in one case.

Table 2: Showing complications associated with limb salvage surgery with endoprosthesis

\begin{tabular}{|c|c|}
\hline Complications & Cases \\
\hline Foot drop & 2 \\
\hline Knee dislocation & 1 \\
\hline Flap necrosis & 1 \\
\hline Aseptic loosening & 1 \\
\hline
\end{tabular}

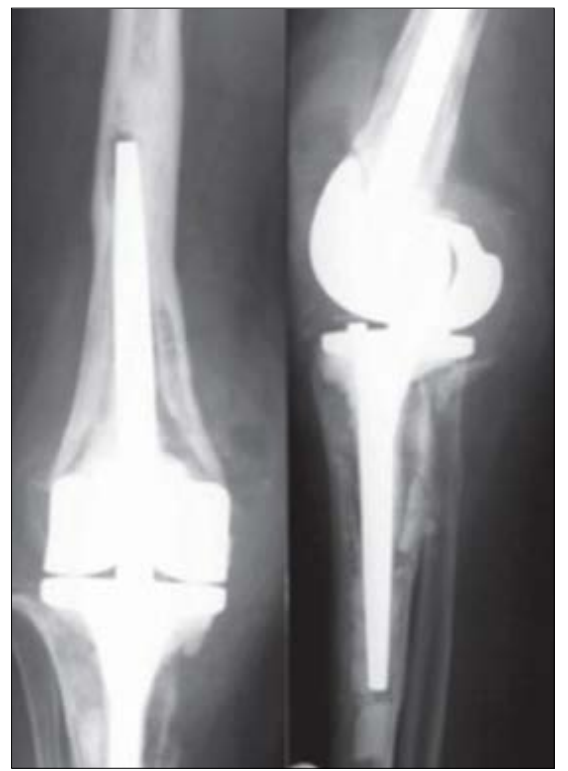

Fig 4: Showing aseptic loosening of the prosthesis following postoperatively at 18 months

\section{Discussion}

Chemotherapy has dramatically changed the cure rate of malignant bone tumours. More than $80 \%$ of patients with sarcoma of an extremity have been considered candidates for limb-salvage surgery following the introduction of effective neoadjuvant chemotherapy ${ }^{[6,7]}$. Individual approach to every patient is essential in order to choose the most suitable surgical treatment. Prosthetic reconstruction in the skeletally immature patient is demanding because of the necessity to cope with the expected disruption of limb growth after resection of one or more major growth plates and the high demands placed on implants by young patients. All our patients were below 30 years and $2 / 3^{\text {rd }}$ were below 25 years of age, hence limb salvage is preferred over amputation.

\section{Conclusion}

Mega endoprosthetic reconstruction provides good functional outcome in patients with bone tumours around the knee. Endoprosthesis should be considered as treatment of choice for bone tumours around the knee. Our study is only a short term follow up and also included wide variety of cases. Hence, long term follow up is necessary to say that the mega endoprosthesis would be a better option to be adopted for limb salvage surgery for sarcomas around knee.

\section{References}

1. Eckardt JJ, Eilber FR, Dorey FJ, Mirra JM. The UCLA experience in limb salvage surgery for malignant tumours. Orthopedics 8 P612-621.

2. Natarajan M, Bose JC, Rajkumar G. Proximal femoral reconstruction with custom mega prosthesis. Int Orthop 2003;27:175-179.

3. Donati D, Giacomini S, Gozzi E, Mercuri M. Proximal femur reconstruction by an allograft prosthesis composite. Clin Orthop 2002;394:192-200.

4. Gibbs CP, Weber K, Scarborough MT. Malignant bone tumours. J Bone Joint Surg (Am) 2001;83:1728-1745.

5. Posinkovic B, Orlic D. Prosthetic replacement of the knee in the treatment of infected and recurring giant cell tumour of the distal femur. Arch Orthop Trauma Surg 1983;102:131-134.

6. Bacci G, Picci P, Ferrari S, Ruggieri P, Casadei R, Tienghi A et al. Primary chemotherapy and delayed surgery for nonmetastatic osteosarcoma of the extremities. Results in 164 patients preoperatively treated with high doses of methotrexate followed by cisplatin and doxorubicin. Cancer 1993;72:3227-3238.

7. Link MP, Goorin AM, Horowitz M, Meyer WH, Belasco J, Baker A et al. Adjuvant chemotherapy of high grade osteosarcoma of the extremity. Updated results of the multi institutional osteosarcoma study. Clin Orthop Relat Res 1991;270:8-14.

8. Weisstein JS, Goldsby RE, O’Donnell RJ. Oncologic approaches to pediatric limb preservation. J Am Acad Orthop Surg 2005;13:544-554.

9. Abudu A, Grimer R, Tillman R, Carter S. The use of prostheses in skeletally immature patients. Orthop Clin North Am 2006;37:75-84.

10. Orlic D, Smerdeji M, Kolundzic R, Bergovec M. Lower limb salvage surgery: Modular rndoprosthesis in bone tumour treatment. International Orthopaedics 2006;30(6):458-464. 